\title{
Hybrid Model for Learner Modelling and Feedback Prioritisation in Exploratory Learning
}

\author{
Mihaela Cocea and George D. Magoulas * \\ London Knowledge Lab, Birkbeck College, University of London, UK
}

\begin{abstract}
Individual and/or hybrid AI techniques are often used in learning environments for well-structured domains to perform learner diagnosis, create and update a learner model and provide support at the individual or group level. This paper presents a conceptual model that employs a synergistic approach based on Case-Based Reasoning (CBR) and Multicriteria Decision Making (MDM) components for learner modelling and feedback generation during exploration in an ill-defined domain of mathematical generalisation. The model uses a CBR component to represent and match the learners' constructions and the strategies adopted during exploratory learning activities. The CBR component is used to diagnose what students are doing on the basis of simple and composite cases that allow a detailed diagnosis of the learners' constructions and facilitate contextualised and personalised feedback. Simple cases represent parts of the models that the learners could possibly construct during an exploratory learning activity, while composite cases, which are assembled from simple cases, correspond to strategies that learners may adopt to construct their models; similarity measures are used to identify how close/far are the learners from solutions pre-specified and stored in the knowledge base. This information is then fed into the MDM component that is responsible for prioritising types of feedback depending on the context. This is based on a formulation of the feedback generation problem in terms of a multicritiria decision making: a set of criteria are considered together with a set of alternatives, the later corresponding to different types of feedback. The operation of the two components and the effectiveness of the synergistic approach are validated through user scenarios in the context of an exploratory learning environment for mathematical generalisation.
\end{abstract}

\section{Introduction}

Intelligent Learning Environments (ILEs) make use of a variety of AI techniques, either in stand alone mode or in combination with others. Among such techniques are: case-based reasoning [59, 19], neural networks [2], Bayesian networks [4, 10], genetic and evolutionary algorithms [51], neuro-fuzzy systems [57], genetic algorithms and

*emails: \{mihaela;gmagoulas\}@dcs.bbk.ac.uk 
case-based reasoning [24], expert systems with genetic algorithms [31], hybrid rules integrating symbolic rules with neurocomputing [20].

Our work focuses on a particular class of ILEs that adopt the principles of discovery learning, which emphasises opportunities to learn through free exploration and discovery rather than guided tutoring. Exploratory Learning Environments (ELEs) are built on this idea and with the principles of constructivism paradigm for teaching and learning in mind. This approach has proved to be beneficial for learners in terms of acquiring deep conceptual and structural knowledge. However, discovery learning without guidance and support appears to be less effective than step-by-step guiding learning environments [27]. To this end, an understanding of learner's behaviour and knowledge construction is needed [44].

Simulations are used in most existing ELEs as a way of actively involving learners in the learning process (e.g. [60], [25]) and cognitive tools [62] are deployed to support their learning. Few such systems model learner's knowledge/skills; for example [4] and [10] use bayesian networks, while [57] combines neural networks with fuzzy representation of knowledge. Another category of ELEs is closer to the constructivist approach by allowing the learner to construct their own models rather than explore a "predefined" one. Compared to conventional learning environments and even environments that use simulations, this type of ELE requires approaches to learner modelling that would be able to capture and model the useful interactions that take place as learners construct their models.

In this paper, we present a conceptual model for learner modelling and feedback generation, which is suitable for ELEs that involve exploring simulations and constructing models. This is based on a hybrid approach that exploits the synergy of case-based reasoning and multicriteria decision making. A novel use of case-based representation is presented, with a different level of information granularity: simple cases represent parts of a problem solution, while composite cases represent different solutions of the same problem. Also, feedback prioritisation is formulated as a multicriteria decision problem and, more specifically, in terms of Analytic Hierarchy Process (AHP), one of the most popular methods of MDM; the AHP is thus used in new context, i.e. feedback generation. Scenarios-based validation is used to assess the behaviour of the model in the context of an exploratory environment for teaching mathematical generalisation.

The rest of the paper is organised as follows. The subsequent section presents a review on hybrid approaches involving the two components of the proposed model, i.e. CBR and MDM. Section 3 introduces the application domain, namely mathematical generalisation, and the ELE used, called ShapeBuilder; it also discusses the challenges involved in the particular application domain. Section 4 describes the modules of the system and discusses how they are organised to support learner modelling and feedback generation. Sections 5 and 6 present the two components of the hybrid model. Section 5 describes the case-based reasoning formalisation in the context of ShapeBuilder and validates the operation of the CBR component through a scenario, whilst Section 6 addresses the multicriteria decision making component and its validation. Section 7 validates the synergy of the two components through a scenario that shows how the two methods are combined and coordinated, and effectively work together. Lastly, Section 8 concludes the paper and outlines our future plan. 


\section{Relevant Work on Synergistic Approaches involving CBR and MDM}

Many real applications use hybrid approaches in order to combine the strengths or compensate for the weaknesses of different methods. Our model combines Case-based Reasoning with the Analytic Hierarchy Process, a method from Multicriteria Decision Making, for learner modelling and feedback generation. This combination of methods has been used for message filtering [35] and bankruptcy prediction [47], and both CBR and AHP are very often used in combination with other methods.

Among others, CBR has been used in various domains (e.g. engineering, manufacturing, medical diagnosis, e-Learning) in combination with other methods like: rule-based approaches [38], association-based case reduction technique [39], fuzzy logic [7, 64], genetic algorithms [24], neural networks [48], statistical methods [9], ontologies [63], model-based reasoning [40], knowledge discovery and data mining [65, 46]. CBR has also been used in combination with more than just one method: generic algorithms and knowledge discovery and data mining [65]; rule-based reasoning and fuzzy logic [5]; neural networks and fuzzy logic [55]; neural networks, fuzzy theory, induction, utility theory, and knowledge-based planning [23].

Mulicriteria Decision Making (MDM) has many applications in fields where decisions need to be taken. The Analytic Hierarchy Process is one of the most popular methods in MDM and is widely applied in a diversity of areas such as logistics [6, 49], military applications [30,11], manufacturing [13, 56], health-care applications [34, 37] and most of the time in combination with other methods [22]. A recent literature review [22] reports five main categories of tools integrated with AHP: (a) mathematical programming, (b) quality function development, (c) meta-heuristics, (d) SWOT analysis and (e) data envelopment analysis. Four works related to higher education are reported in areas of IT-based project selection [33], teaching method selection [36], education requirement selection [28] and faculty course assignment [45].

In the area of learner/user modelling, AHP has been used in combination with fuzzy logic [16] for student diagnosis in an adaptive hypermedia educational system and in combination with Multi-Attribute Utility Theory (MAUT), another method from MDM, in recommender systems [53], where the evaluation function from MAUT is used to rate how well each alternative fulfills the decision criteria.

The following sections present the domain of mathematical generalisation and the ELE used in our work, called ShapeBuilder.

\section{Exploratory Learning for Mathematical Generalisa- tion}

Mathematical generalisation (MG) is associated with algebra, as "algebra is, in one sense, the language of generalisation of quantity. It provides experience of, and a language for, expressing generality, manipulating generality, and reasoning about generality" [41].

However, students do not associate algebra with generalisation as the algebraic 
language is perceived as been separate from what it represents [26]. To address this problem the ShapeBuilder system [17, 50], which is an ELE developed in the context of the MiGen project ${ }^{1}$, aims to facilitate the correspondence between the models, patterns and structures (visual representations) that the learners build, on one hand, and their numeric, iconic and symbolic representations, on the other hand. ShapeBuilder (see Figure 1) allows the construction of different shapes [18], e.g. rectangles, L-shapes, T-shapes and supports the three types of representations aforementioned: (a) numeric representations that include numbers (constants or variables) and expressions with numbers; (b) iconic representations which correspond to icon variables; (c) symbolic representations that are names or symbols given by users to variables or expressions. An icon variable has the value of a dimension of a shape (e.g. width, height) and can be obtained by double-clicking on the corresponding edge of the shape. It is represented as an icon of the shape with the corresponding edge highlighted (see Figure 2a).

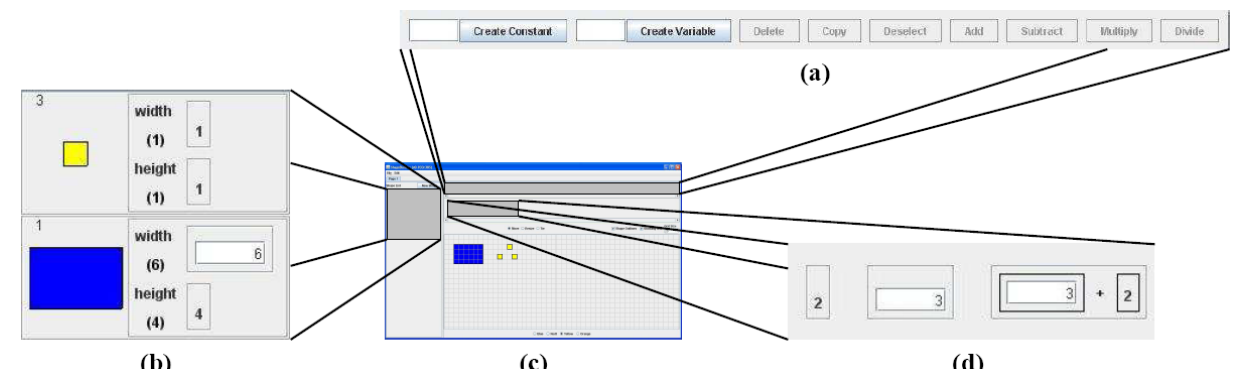

(b)

(c)

(d)

Figure 1: The layout of the ShapeBuilder. (a) the Expression Toolbar; (b) the Shape List; (c) the overall ShapeBuilder interface (the gridded area is the interaction canvas); (d) the Expression Palette. The figure is reproduced from [17].

Constants, variables and numeric expressions lead to specific constructions/models, while icon variables and expressions using them lead to general ones. Through the use of icon variables, ShapeBuilder encourages structured algebra thinking, connecting the visual with the abstract (algebraic) representation, as "each expression of generality expresses a way of seeing" [41] (see Figure 2b). It also uses the "messing up" metaphor [21] that consists of asking the learner to resize a construction and observe the consequences; the model will "mess up" only if it is not general (see Figures $2 \mathrm{c}$ and d), indicating learner's lack of generalisation ability.

In the development of the system two phases of the cooperative inquiry design [12] were used: (a) a contextual inquiry phase, when data was collected in the user's environment (in our case, classrooms), and (b) a participatory design phase, when teachers were involved in the design of prototypes and children tested the developed prototypes. Data from both phases was used in subsequent design iterations and informed further development decisions. User-centred iterative approaches, like the one followed in

\footnotetext{
${ }^{1}$ Funded by ESRC, UK, under TLRP e-Learning Phase-II (RES-139-25-0381); http : / www . t l rp. org/proj/tel/tel_noss.html.
} 
the design of ShapeBuilder, take more development time compared to other software development methodologies [15].

When attempting to model the learner and provide feedback in an ELE for such a wide domain as MG, several challenges arise. The main and widely acknowledged challenge is to balance freedom with control: learners should be given enough freedom so that they can actively engage in activities but they should be offered enough guidance and feedback in order to assure that the whole process reflects constructivist learning and leads to useful knowledge [43]. This and some other challenges are summarised in Table 1 with examples from the domain of mathematical generalisation.
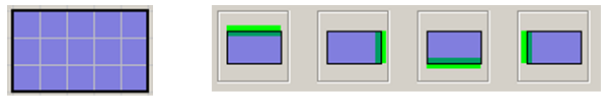

(a)

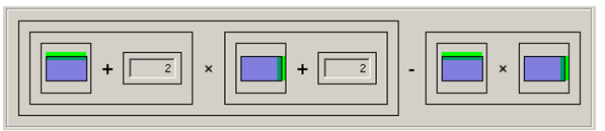

(b)

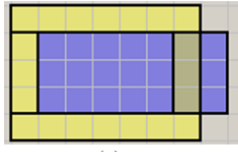

(c)

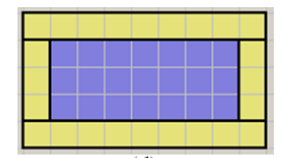

(d)

Figure 2: (a) A rectangular shape and its icon variable; (b) an expression using icon variables; (c) "messing up"; (d) general solution that does not "mess up".

Given the challenges mentioned in Table 1 a conventional learner modelling approach does not fit the purposes of ELEs. Due to the exploratory nature of the activities and the diversity of possible trajectories, flexibility in the representation of information and handling multiple feedback are two important aspects for effectively supporting the learning process. As case-based reasoning offers flexibility of information representation and multicriteria decision making techniques handle prioritization of alternatives, a combination of the two is used. The following sections presents the overall architecture and the components of the hybrid model.

\section{An Architecture for Learner Modelling and Feedback Generation}

The architecture of the proposed model and its relationships with the ShapeBuilder environment are represented in Fig. 3. As the learner interacts with the system through the interface, the actions of the learner are stored in the Short-Term (STM) part of the Learner Model (LM) and are passed to the Interactive Behaviour Analysis Module (IBAM) where they are processed in cooperation with the Task Model from the Knowledge Base (KB); the results are fed into the Task Long-Term (LTM) part of the learner model. The Feedback Module (FM) is informed by the LM and the KB and feeds back 
Table 1: Challenges for learner modelling and feedback in exploratory learning for mathematical generalisation.

\begin{tabular}{ll}
\hline $\begin{array}{l}\text { Challenge } \\
\text { Balance between freedom } \\
\text { and control }\end{array}$ & $\begin{array}{l}\text { Whample a learner is trying to produce a general represen- } \\
\text { tation, for how long should he be left alone to explore } \\
\text { and when does feedback become necessary? }\end{array}$ \\
\hline What should be modelled? & $\begin{array}{l}\text { Besides learner's knowledge of MG concepts (e.g. use } \\
\text { of variables, consistency between representations, etc.), } \\
\text { other aspects need to be modelled in order to support the } \\
\text { learner during exploration: shapes constructed, relations } \\
\text { between shapes, etc. }\end{array}$ \\
\hline $\begin{array}{l}\text { Do both correct and incor- } \\
\text { rect learner actions or be- }\end{array}$ & $\begin{array}{l}\text { In exploratory learning it is difficult to categorise actions } \\
\text { or learner's explorations into "correct" and "incorrect". } \\
\text { haviours have value? }\end{array}$ \\
$\begin{array}{l}\text { Moreover, actions that might lead to incorrect outcomes } \\
\text { such as resizing can be more valuable for constructivist } \\
\text { learning than "correct" actions. }\end{array}$ \\
$\begin{array}{l}\text { Reasoning about abstract } \\
\text { knowledge }\end{array}$ & $\begin{array}{l}\text { Can consistency be inferred from the fact that a learner } \\
\text { is checking the correspondence between various forms } \\
\text { of representations? If so, is that always true? Are there } \\
\text { any exceptions to this rule? }\end{array}$ \\
\hline $\begin{array}{l}\text { As it is neither realistic nor feasible to include all possi- } \\
\text { ble outcomes (correct or incorrect) to model the domain } \\
\text { of MG, only key information with educational value } \\
\text { could be stored, such as strategies in solving a task. The } \\
\text { challenge is how to represent and detect them. }\end{array}$ \\
\hline
\end{tabular}


to the learner through the interface. A part of the FM, the Feedback Priorities (FP) component which is responsible for handling multiple feedback, is informed by the Task LTM and Domain LTM of the learner model (the double-line arrows in Fig. 3).

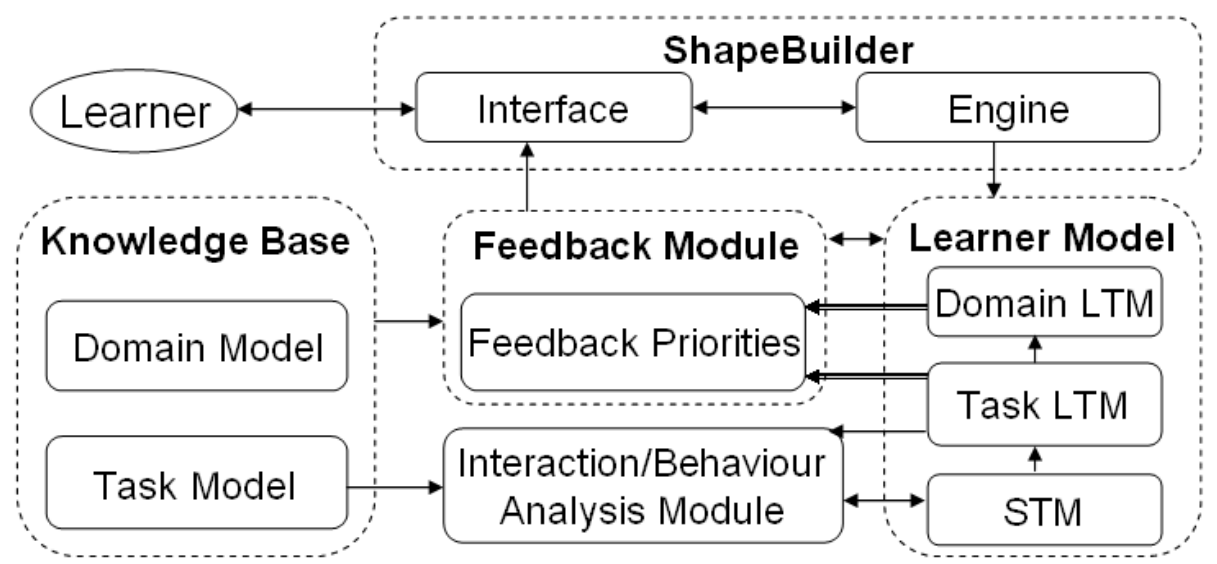

Figure 3: Intelligent components and their interrelationships.

The KB includes two components (see Figure 3): a domain and a task model. The domain model includes high level learning outcomes related to the domain (e.g. using variables, structural reasoning, consistency, etc.) and considers that each learning outcome can be achieved by exploring several tasks. The task model includes different types of information: (a) strategies of approaching the task which could be correct, incorrect or partially correct; (b) outcomes of the exploratory process and solutions to specific questions associated with each (sub)task; (c) landmarks, i.e. relevant aspects or critical events occurring during the exploratory process; (d) contexts, i.e. reference to particular (sub)tasks.

The Learner Model has three components: (a) a Short-Term Model where the recent actions of the learner are stored and passed to the IBAM component; (b) a Task LongTerm Model that contains information about the tasks performed by the learner and which is updated via the IBAM; (c) a Domain Long-Term model which is an overlay model of the Domain Model.

The IBAM component uses case-based reasoning to identify what learners are doing (having information from the STM component of the LM) and be able to inform the feedback module as they explore a (sub)task. More specifically, as they are working in a specific subtask, which specifies a certain context, their actions are preprocessed, current cases are identified and matched to the cases from the Task Model (the case base), and this information is passed to the Task LTM.

Multicriteria decision making [14] is used in the Feedback Priorities component of the Feedback Module to obtain priorities between several aspects that require feedback depending on the current (sub)task and context (Task LTM) and on user's characteristics (Domain LTM). 


\section{The Case-Based Reasoning Component}

In CBR [29] the knowledge is stored as cases, typically including the description of a problem and the corresponding solution. When a new problem is encountered, similar cases are searched and the solution is adapted from one or more of the most similar cases.

Although CBR has been used successfully in applications for domains like legal reasoning [1], stock market prediction [8], recommender systems [32], and other areas, there is little research on using CBR for e-Learning environments. For example, [19] uses CBR in the learner modelling process and call this approach case-based student modelling; while [24] uses CBR and genetic algorithms to construct an optimal learning path for each learner. CBR is used also in [59] within a case-based instruction scenario rather than a method for learner modelling. To the best of our knowledge, there is no previous work in the area of ELEs that use CBR or CBR combined with other intelligent methods.

The advantage of CBR for learning environments and especially for ELEs is that the system does not rely only on explicit representation of general knowledge about a domain, but it can also use specific knowledge previously experienced [19]. It also seems promising for improving the effectiveness of complex and unstructured decision making [24] in combination with other computing methods.

\subsection{Case-based Knowledge Representation}

In our research, the cases contain information describing models that learners could construct using ShapeBuilder. Different strategies in approaching a problem (i.e. constructing a model to meet a particular learning objective) are represented as a series of cases that reflect possible exploratory trajectories of learners as they construct models during the various (sub-)tasks.

The cases were built using input from the contextual and the participatory phase of the design methodology described in Section 3. The collected data (i.e. log files, notes, videos) was analysed and synthesized, leading to the knowledge representation presented below. Learner-centred iterative approaches, like the one adopted in our work for eliciting the information represented and designing the cases, are naturally more time consuming than standard development methodologies [15].

A case is defined as $C_{i}=\left\{F_{i}, R A_{i}, R C_{i}\right\}$, where $C_{i}$ represents the case and $F_{i}$ is a set of attributes. $R A_{i}$ is a set of relations between attributes and $R C_{i}$ is a set of relations between $C_{i}$ and other cases respectively.

The set of attributes is represented as $F_{i}=\left\{\alpha_{i_{1}}, \alpha_{i_{2}}, \ldots, \alpha_{i_{N}}\right\}$. It includes three types of attributes: (a) numeric, (b) variables and (c) binary. Variables refer to different string values that an attribute can take, and binary attributes indicate whether a case can be considered in formulating a particular strategy or not. This could be represented as a "part of strategy" function: PartOf $S_{u}: C_{i} \rightarrow\{0,1\}$,

$$
\operatorname{PartOfS}_{u}= \begin{cases}1 & \text { if } C_{i} \in S_{u} \\ 0 & \text { if } C_{i} \notin S_{u}\end{cases}
$$


where $S_{u}$ represents a strategy and is defined further on. The set of attributes of a generic case for ShapeBuilder is presented in Table 2. The first $v$ attributes $\left(\alpha_{i_{j}}, j=\right.$ $\overline{1, v})$ are variables, the ones from $v+1$ to $w$ are numeric $\left(\alpha_{i_{j}}, j=\overline{v+1, w}\right)$ and the rest are binary $\left(\alpha_{i_{j}}, j=\overline{w+1, N}\right)$.

Table 2: The set of attributes $\left(F_{i}\right)$ of a case.

\begin{tabular}{lccc}
\hline Category & Name & Label & Possible Values \\
\hline Shape & Shape type & $\alpha_{i_{1}}$ & Rectangle(/L-Shape/T-Shape) \\
\hline $\begin{array}{l}\text { Dimensions } \\
\text { of shape }\end{array}$ & Width type & $\alpha_{i_{2}}$ & $\begin{array}{c}\text { constant (c)/variable (v)/ } \\
\text { icon variable (iv)/ } \\
\text { numeric expression (n_exp)/ } \\
\text { expression with iv(s) (iv_exp) }\end{array}$ \\
\cline { 2 - 4 } & Height type & $\alpha_{i_{3}}$ & c /v /iv /n_exp /iv_exp \\
\cline { 2 - 4 } & $\vdots$ & $\vdots$ & $\vdots$ \\
\cline { 2 - 4 } & Thickness type & $\alpha_{i_{v}}$ & c /v /iv /n_exp /iv_exp \\
\cline { 2 - 4 } & Width value & $\alpha_{i_{v+1}}$ & numeric value \\
\cline { 2 - 4 } & Height value & $\alpha_{i_{v+2}}$ & numeric value \\
\cline { 2 - 4 } Strategy & $\vdots$ & $\vdots$ & $\vdots$ \\
\cline { 2 - 4 } & Thickness value & $\alpha_{i_{w}}$ & c /v /iv /n_exp /iv_exp \\
\cline { 2 - 4 } & PartOf $S_{1}$ & $\alpha_{i_{w+1}}$ & 1 \\
\cline { 2 - 4 } & PartOf $S_{2}$ & $\alpha_{i_{w+2}}$ & 0 \\
\cline { 2 - 4 } & $\vdots$ & $\vdots$ & $\vdots$ \\
\cline { 2 - 4 } & PartOf $S_{r}$ & $\alpha_{i_{N}}$ & 0 \\
\hline
\end{tabular}

The set of relations between attributes of the current case and attributes of other cases (as well as attributes of the same case, if there are any present) is represented as $R A_{i}=\left\{R A_{i_{1}}, R A_{i_{2}}, \ldots, R A_{i_{M}}\right\}$, where at least one of the attributes in each relation $R A_{i_{m}}, \forall m=\overline{1, M}$, is from the set of attributes of the current case $F_{i}$. Two types of binary relations are used: (a) a dependency relation $\left(D_{i_{s}}\right)$ is defined as $\left(\alpha_{i_{k}}, \alpha_{j_{l}}\right) \in$ $D_{i_{s}} \Leftrightarrow \alpha_{i_{k}}=D E P\left(\alpha_{j_{l}}\right)$, where DEP : $\alpha_{i_{k}} \rightarrow \alpha_{j_{l}}$ for attributes $\alpha_{i_{k}}$ and $\alpha_{j_{l}}$ that are variables of cases $i$ and $j$ (where $i=j$ or $i \neq j$ ), and means that $\alpha_{i_{k}}$ depends on (is built upon) $\alpha_{j_{l}}$ (if $i=j, k \neq l$ is a condition as to avoid circular dependencies) (e.g. the width type of a case is built upon the height type of the same case; the width type of a case is built upon the width type of another case, an so on); (b) a value relation $\left(V_{i_{s}}\right)$ is defined as $\left(\alpha_{i_{k}}, \alpha_{j_{l}}\right) \in V_{i_{s}} \Leftrightarrow \alpha_{i_{k}}=f\left(\alpha_{j_{l}}\right)$, where $\alpha_{i_{k}}$ and $\alpha_{j_{l}}$ are numeric attributes and $f$ is a function and could have different forms depending on context (e.g. the height of a shape is two times its width; the width of a shape is three times the height of another shape, etc.). The set of relations between attributes is presented in Table 3.

The set of relations between cases is represented as $R C_{i}=\left\{R C_{i_{1}}, R C_{i_{2}}, \ldots, R C_{i_{P}}\right\}$, where one of the cases in each relation $R C_{i_{j}}, \forall j=\overline{1, P}$ is the current case $\left(C_{i}\right)$. Two relations about order in time are defined: (a) Prev relation indicates the previ- 
Table 3: The set of relations between attributes $\left(R A_{i}\right)$ of cases.

\begin{tabular}{lcc}
\hline Relation & Label & Example \\
\hline Dependency relation & $D_{i_{1}}\left(R A_{i_{1}}\right)$ & $\left(\alpha_{i_{k}}, \alpha_{j_{l}}\right) ; k, l=\overline{2, v} ; \forall j$ \\
\cline { 2 - 3 } & $\vdots$ & $\vdots$ \\
\cline { 2 - 3 } Value relation & $D_{i_{t}}\left(R A_{i_{t}}\right)$ & $\left(\alpha_{i_{k}}, \alpha_{j_{l}}\right) ; k, l=\overline{2, v ; \forall j}$ \\
\cline { 2 - 3 } & $V_{i_{1}}\left(R A_{i_{t+1}}\right)$ & $\left(\alpha_{i_{k}}, \alpha_{j_{l}}\right) ; k, l=\overline{v+1, w ; \forall j}$ \\
\cline { 2 - 3 } & $\vdots$ & $\vdots$ \\
\cline { 2 - 3 } & $V_{i_{z}}\left(R A_{i_{M}}\right)$ & $\left(\alpha_{i_{k}}, \alpha_{j_{l}}\right) ; k, l=\overline{v+1, w ; \forall j}$ \\
\hline
\end{tabular}

ous case with respect to the current case: $\left(C_{i}, C_{j}\right) \in \operatorname{Prev}$ if $t\left(C_{j}\right)<t\left(C_{i}\right)$ and (b) Next relation indicates the next case with respect to the current case: $\left(C_{i}, C_{k}\right) \in$ $N$ ext if $t\left(C_{i}\right)<t\left(C_{k}\right)$. Each case includes at most one of each of these two relations $(p \leq 2)$.

A strategy is defined as $S_{u}=\left\{N_{u}(C), N_{u}(R A), N_{u}(R C)\right\}, u=\overline{1, r}$, where $N_{u}\left(C_{i}\right)$ is a set of cases, $N_{u}\left(R A_{i}\right)$ is a set of relation between attributes of cases and $N_{u}\left(R C_{i}\right)$ is a set of relations between cases.

\subsection{Comparing Cases}

The most common definition of similarity is a weighted sum of similarities of attributes of cases [29]:

$$
S_{I R}=\frac{\sum_{i=1}^{N} o_{i} \times \operatorname{sim}\left(f_{i}^{I}, f_{i}^{R}\right)}{\sum_{i=1}^{n} o_{i}}
$$

where $o_{i}$ represents the weight of each attribute, sim is a similarity function, and $I$ and $R$ stand for input and retrieved cases, respectively. In our case, four similarity measures are defined for comparing cases:

1. Euclidean distance is used for comparing numeric attributes:

$$
D_{I R}=\sqrt{\sum_{j=v+1}^{w} o_{j} \times\left(\alpha_{I_{j}}-\alpha_{R_{j}}\right)^{2}}
$$

2. The following metric is used for attributes that are variables: $V_{I R}=\frac{\sum_{j=1}^{v} g\left(\alpha_{I_{j}}, \alpha_{R_{j}}\right)}{v}$, where $g$ is defined as:

$$
g\left(\alpha_{I_{j}}, \alpha_{R_{j}}\right)= \begin{cases}1 & \text { if } \alpha_{I_{j}}=\alpha_{R_{j}} \\ 0 & \text { if } \alpha_{I_{j}} \neq \alpha_{R_{j}}\end{cases}
$$

3. In a similar way to [54], we define the following metric for comparing relations between attributes: $P_{I R}=\frac{\left|R A_{I} \cap R A_{R}\right|}{\left|R A_{I} \cup R A_{R}\right|}$. $P_{I R}$ is the number of relations between attributes that the input and retrieved case have in common divided by the total number of relations between attributes of the two cases. 
4. Similarity in terms of relations between cases is defined by $T_{I R}=\frac{\left|R C_{I} \cap R C_{R}\right|}{\left|R C_{I} \cup R C_{R}\right|}$, where $T_{I R}$ is the number of relations between cases that the input and retrieved case have in common divided by the the total number of relations between cases of $I$ and $R$.

In order to identify the closest strategy to the one employed by a learner, cumulative similarity measures are used for each of the four types of similarity:

1. Numeric attributes - as this metric has a reversed meaning compared to the other ones, i.e. a smaller number means a greater similarity, the following function is used to bring it to the same meaning as the other three similarity measures, i.e. a greater number means greater similarity.

$$
F_{1}=\left\{\begin{aligned}
\frac{z}{\sum_{i=1}^{z} D_{I_{i} R_{i}}} & \text { if } \sum_{i=1}^{z} D_{I_{i} R_{i}} \neq 0 \\
z & \text { if } \sum_{i=1}^{z} D_{I_{i} R_{i}}=0
\end{aligned}\right.
$$

2. Variables: $F_{2}=\left(\sum_{i=1}^{z} V_{I_{i} R_{i}}\right) / z$.

3. Relations between attributes: $F_{3}=\left(\sum_{i=1}^{z} P_{I_{i} R_{i}}\right) / z$.

4. Relations between cases. $F_{4}=\left(\sum_{i=1}^{z} T_{I_{i} R_{i}}\right) / z$.

where $z$ represents the minimum number of cases among the two compared strategies. The strength of similarity between the current strategy and the various stored strategies is defined as the maximum combined similarity of these four measures among the various strategies compared.

\subsection{Scenario-based Validation of the CBR component}

To illustrate the operation of the CBR component and its role to support learner modelling we use an example from ShapeBuilder, a task called "pond tiling", which is common in the English school curriculum and expects learners to produce a general expression for finding out how many tiles are required for surrounding any rectangular pond [17]. The high level learning objective in the Domain Model is to acquire the ability to perform structural reasoning [18]. In order to achieve this, sub-tasks can be explored in ShapeBuilder, e.g. construct a pond of fixed dimensions, surround the pond with tiles and determine how many are required; generalise the structure using icon variables.

\subsubsection{Representation of Users' Strategies}

The Task Model for pond tiling includes: (a) strategies identified in pilot studies [18], e.g. thinking in terms of areas (see Fig. 4a) or in terms of width and height (see Figures 4b, c, d, e and f); (b) outcomes, e.g. model built, number of tiles for surrounding a particular pond, and solution, i.e. the general expression (see Fig. 4 for the solutions corresponding to each strategy; for the "area strategy" the solution with icon variables is displayed in Fig. 2b); (c) landmarks, e.g. for the area strategy: creating a rectangle 
with height and width greater than the pond by 2 tiles; for the width and height strategies: using rows/column of tiles; slips: several correct actions followed by an incorrect one (e.g. correct surrounding of the pond, partially correct expression, but missing a 2 in the formula); (d) the context of each (sub-)task.

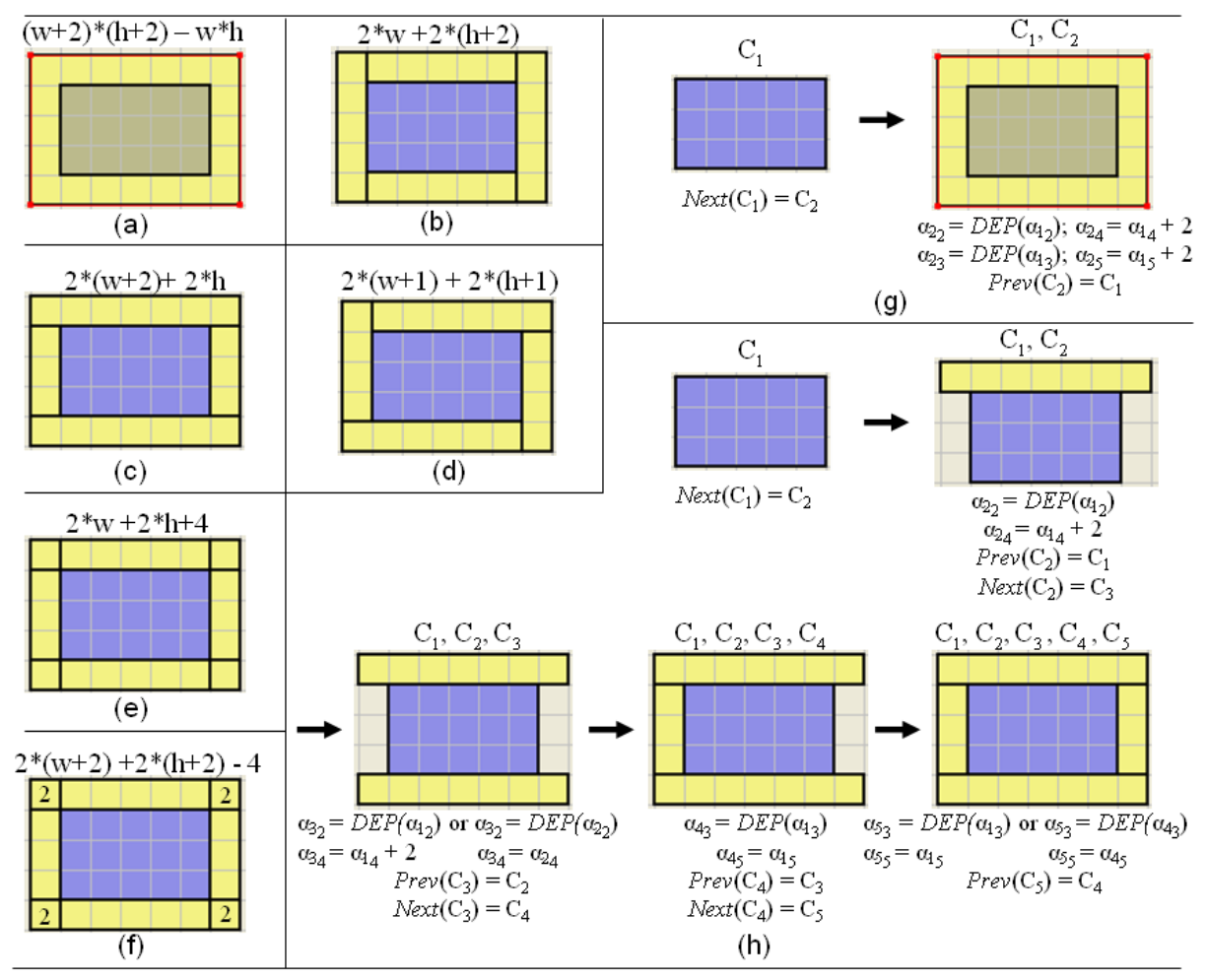

Figure 4: (a) "Area strategy"; (b) "H strategy"; (c) "I strategy"; (d) "Spiral strategy"; (e) "+4 strategy"; (f) "- 4 strategy"; (g) Steps and relations of "area strategy"; (h) Steps and relations of "I strategy".

The six strategies and their associated solutions (the general expressions for surrounding any rectangular pond) are displayed in Figures 4(a-f). Two strategies are presented in detail: the "area strategy" $\left(S_{1}\right)$ and the "I strategy" $\left(S_{3}\right)$. The attributes of cases that are part of these two strategies are presented in Table 4 and Table 5, respectively. The steps and the sets of relations between attributes and between cases are displayed in Figure 4g and Figure 4h, respectively.

A particular order between cases is presented for the "I strategy" in Figure 4h. For the same strategy, the surrounding of the pond could be done in several other different orders; there are $4 !=24$ such possibilities (the pond is always first).

There are two types of strategies depending on the degree of generality: specific and general. Specific cases refer to surroundings that cannot be generalised and include value relations, but no dependency relations; the general cases refer to surround- 
Table 4: The set of attributes $\left(F_{i}\right)$ for the cases in the "area strategy".

\begin{tabular}{lccc}
\hline Name & Label & $C_{1}$ & $C_{2}$ \\
\hline Shape type & $\alpha_{i_{1}}$ & Rectangle & Rectangle \\
\hline Width type & $\alpha_{i_{2}}$ & c/v/n_exp & iv/iv_exp \\
\hline Height type & $\alpha_{i_{3}}$ & c/v/n_exp & iv/iv_exp \\
\hline Width value & $\alpha_{i_{4}}$ & 5 & 7 \\
\hline Height value & $\alpha_{i_{5}}$ & 3 & 5 \\
\hline PartOf $S_{1}$ & $\alpha_{i_{6}}$ & 1 & 1 \\
\hline$\vdots$ & $\vdots$ & $\vdots$ & $\vdots$ \\
\hline PartOf $S_{2}$ & $\alpha_{i_{7}}$ & 1 & 0 \\
\hline PartOf $S_{6}$ & $\alpha_{i_{8}}$ & 1 & 0 \\
\hline
\end{tabular}

Table 5: The set of attributes $\left(F_{i}\right)$ for the cases in the "I strategy".

\begin{tabular}{lccccc}
\hline Label & $C_{1}$ & $C_{2}$ & $C_{3}$ & $C_{4}$ & $C_{5}$ \\
\hline$\alpha_{i_{1}}$ & Rectangle & Rectangle & Rectangle & Rectangle & Rectangle \\
\hline$\alpha_{i_{2}}$ & c/v/n_exp & iv /iv_exp & iv /iv_exp & c/v/n_exp & c/v/n_exp \\
\hline$\alpha_{i_{3}}$ & c/v/n_exp & c/v/n_exp & c/v/n_exp & iv /iv_exp & iv /iv_exp \\
\hline$\alpha_{i_{4}}$ & 5 & 7 & 7 & 1 & 1 \\
\hline$\alpha_{i_{5}}$ & 3 & 1 & 1 & 3 & 3 \\
\hline$\alpha_{i_{6}}$ & 1 & 0 & 0 & 0 & 0 \\
\hline$\alpha_{i_{7}}$ & 1 & 1 & 1 & 1 & 1 \\
\hline$\vdots$ & $\vdots$ & $\vdots$ & $\vdots$ & $\vdots$ & $\vdots$ \\
\hline$\alpha_{i_{8}}$ & 1 & 0 & 0 & 1 & 1 \\
\hline
\end{tabular}

ings that can be generalised and are distinguished by the presence of the dependency relations and by the fact that the dimension type of at least one of the dimensions of the case is an icon variable or an expression using icon variable(s). The presence or absence of the abovementioned aspects apply to all cases that form the composite case with the exception of the first case representing the pond. The "area" and the "I strategy" presented previously fall into the category of general strategies.

The strategies displayed in Figure 4 are correct symmetrical "elegant" solutions, but trials with pupils have shown that not all of them use this type of approach $[17,18]$. Some pupils surround the pond in a non-systematic manner and with variable degrees of symmetry. Such examples are illustrated in Figure 5.

\subsubsection{Comparing Cases}

To illustrate the operation of similarity measures we use two 'general' non-symmetrical examples of surrounding the pond, displayed in Figure 5 and two examples combining 'specific' and 'general' cases, displayed in Figure 6. The similarity measures are the 
ones presented in Section 5.2. The scenarios correspond to real-life situations encountered in trials with pupils.

Scenario 1. The example displayed in Figure 5a has 4 cases in common with two strategies: the "I strategy" $\left(C_{1}, C_{3}, C_{4}, C_{5}\right)$ and the "+4 strategy" $\left(C_{1}, C_{4}, C_{5}, C_{6}\right)$. When comparing it with the "I strategy" $z=5$ (minimum between 6 and 5) and the combined similarity is: $\frac{5}{\sqrt{1}}+\frac{5}{5}+\frac{7 / 4}{5}+\frac{10 / 4}{5}=6.85$. When comparing with the " +4 " strategy, $z=6$ (minimum between 6 and 9) the combined similarity is: $\frac{6}{\sqrt{5}}+\frac{5+2 / 3}{6}+$ $\frac{6 / 4}{6}+\frac{10 / 4+1 / 3}{6}=4.35$. Thus, in this case the learner will be guided towards the "I strategy".

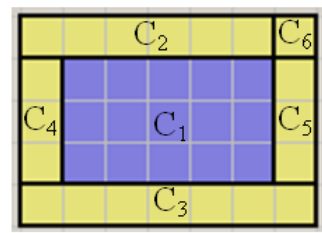

(a)

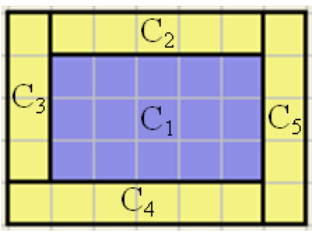

(b)

Figure 5: Non-symmetrical strategies: (a) combination of 'I' and ' +4 ' strategies; (b) combination of 'spiral' and ' $\mathrm{H}$ ' strategies.

Scenario 2. The second example (Figure 5b), has 3 cases in common with two strategies: the "spiral strategy" $\left(C_{1}, C_{3}, C_{4}\right)$ and the "H strategy" $\left(C_{1}, C_{2}, C_{5}\right)$. When comparing it with the "spiral strategy" as well as the "H strategy", $z=5$ (minimum between 5 and 5), and the combined similarity is: $\frac{5}{\sqrt{2}}+\frac{4+2 / 3}{5}+\frac{8 / 4}{5}+\frac{10 / 4}{5}=5.37$. In this situation, when the learner's construction is equally similar to two strategies, the following options could be offered: (a) present the learner with the two options and let him/her choose one of the two (an approach that appears more suitable for advanced learners than novices); (b) automatically suggest one of the two in a systematic way, e.g. present the one that occurs more/less often with other learners; (c) inform the teacher about the learner's trajectory and the frequency of strategies and let him/her decide between the two.

Scenario 3. The surrounding of the pond in Figure 6a corresponds to the '-4' strategy and has two 'general' cases $\left(C_{2}\right.$ and $\left.C_{3}\right)$ and two 'specific' ones $\left(C_{3}\right.$ and $\left.C_{4}\right)$. When compared to the 'specific' '-4' strategy and to the 'general' '-4' strategy, the results are the same: $5+\frac{3+2 / 3}{5}+\frac{1+2 / 3}{5}+\frac{10 / 4}{5}=6.70$. This is due to the symmetry of the surrounding ( 2 general and 2 specific cases). However, when there is no such symmetry, the results will vary when the surrounding is compared to the 'specific' and to the 'general' strategies. The decision to compare the surrounding of the pond to one of the two types of strategies is made depending on the users characteristics. Generally, if the learner has parts of the surrounding built in a general way and there is no more 
information about the learner's experience with similar tasks or his/her overall level of generalisation ability, the comparison will be made to the 'general' strategies, as some learners are able to generalise from the beginning, without going through a 'specific' phase. However, some learners may copy what their colleagues are doing without really understanding and without being able to generalise their solution. Further activity will reveal whether this is the case, e.g. a learner typically will stack as the colleague he/she copied from is way more advanced in the task; they, however, cannot follow any more as they try to follow up using 'specific' cases. If so, the comparison will be made with the 'specific' strategies and the learners will be supported to achieve a correct surrounding of the pond and a correct expression of the number of tiles surrounding the pond before moving on to generalise.

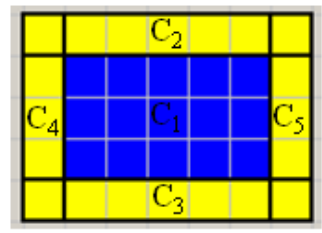

(a)

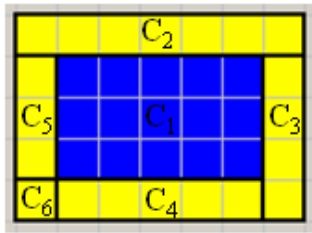

(b)

Figure 6: Strategies combining specific and general cases: (a)'-4' strategy $\left(C_{2}\right.$ and $C_{3}$ are general; $C_{4}$ and $C_{5}$ are specific); (b) combination of ' +4 ', 'spiral' and 'I' strategies $\left(C_{2}\right.$ and $C_{4}$ are general; $C_{3}, C_{5}$ and $C_{6}$ are specific).

Scenario 4. The surrounding displayed in Figure $6 \mathrm{~b}$ is a combination of three strategies (with at least two corresponding cases): ' +4 ', 'spiral' and 'I' strategies; two cases are general $\left(C_{2}\right.$ and $\left.C_{4}\right)$ and three are specific $\left(C_{3}, C_{5}\right.$ and $\left.C_{6}\right)$. Table 6 displays the similarity measures for all three strategies in the 'specific' and 'general' case.

Table 6: Comparisons of Figure $6 \mathrm{c}$ with the specific and general ' +4 ', 'spiral' and 'I' strategies.

\begin{tabular}{l|c|cccc|c}
\hline Strategy type & Strategy & $F_{1}$ & $F_{2}$ & $F_{3}$ & $F_{4}$ & Total \\
\hline \multirow{3}{*}{ Specific } & '+4' & $\frac{6}{\sqrt{12}}$ & $\frac{4+4 / 3}{6}$ & $\frac{1 / 3+1 / 2}{6}$ & $\frac{12 / 4}{6}$ & 3.26 \\
\cline { 2 - 7 } & 'spiral' & $\frac{5}{\sqrt{12}}$ & $\frac{3+4 / 3}{5}$ & $\frac{1 / 2}{5}$ & $\frac{10 / 4+1 / 3}{5}$ & 2.88 \\
\cline { 2 - 7 } & 'I' & $\frac{5}{\sqrt{12}}$ & $\frac{3+4 / 3}{5}$ & $\frac{1+1 / 3}{5}$ & $\frac{10 / 4+1 / 3}{5}$ & 3.04 \\
\hline \multirow{2}{*}{ General } & '+4' & $\frac{6}{\sqrt{12}}$ & $\frac{4+1 / 3}{6}$ & $\frac{3 / 4+1 / 3}{6}$ & $\frac{12 / 4}{6}$ & 3.30 \\
\cline { 2 - 7 } & 'spiral' & $\frac{5}{\sqrt{12}}$ & $\frac{3+4 / 3}{5}$ & $\frac{1 / 2+1 / 3}{5}$ & $\frac{10 / 4+1 / 3}{5}$ & 2.94 \\
\cline { 2 - 7 } & 'I' & $\frac{5}{\sqrt{12}}$ & $\frac{3+4 / 3}{5}$ & $\frac{3 / 4+1 / 3}{5}$ & $\frac{10 / 4+1 / 3}{5}$ & 2.99 \\
\hline
\end{tabular}

As pointed out before, when there is no symmetry with respect to the 'specific' and 'general' cases used in surrounding the pond, the similarity measures are different when compared to the 'specific' and 'general' strategies, as it can be observed in Table 6. Although the maximum similarity measure is obtained for the ' +4 ' strategy, it may 
be easier to guide the student to the 'I' strategy, as the ' +4 ' strategy is more complex, especially if the learner has little experience with tasks of similar difficulty; another reason to 'favour' other strategies over the ' +4 ' one is that learners often complete the surrounding of the pond with ' 1 by 1' tiles just because there are some spaces that need to be completed.

\section{The Multicriteria Decision Making Component}

Multicriteria Decision Making (MDM) defines a class of problems where a decision from a predefined set of alternatives needs to be reached by taking into account two or more criteria. Each alternative is evaluated on the set of criteria and the outcomes provide a means of comparison between the alternatives that will facilitate a selection of one or some alternatives, or a ranking between them. MDM also includes classification of alternatives into groups and group ranking [66]. The various approaches used in this context to solve decision making problems include statistical techniques, multi-attribute utility analysis, the analytic hierarchy process, knowledge bases, and mathematical models.

The Analytic Hierarchy Process (AHP) [52] is one of the most popular methods in MDM [61, 58] that has been successfully applied in different domains, including finance, engineering, sports, and others. It has also been applied in education, as mentioned in Section 2, but not in the area of learner modeling and feedback generation. The next section includes a description of AHP formulation, followed by an example of its application in the context of exploratory learning for mathematical generalisation.

\subsection{The Analytic Hierarchy Process Formalism for Feedback Pri- oritisation}

The Analytic Hierarchy Process uses a hierarchical or network structure to represent a decision problem and to establish priorities between alternatives depending on a set of criteria involved in the decision process. It includes three main steps: (a) construction of the hierarchy; (b) analysis of priorities and (c) verification of consistency.

The process (see Figure 7) starts with the definition of the hierarchy and is followed by several sets of pairwise comparisons for the criteria involved and the possible alternatives. These result in weights for the criteria and priority vectors for the alternatives; their consistency is verified and they are used to calculate the composite weights of alternatives or final priorities; the consistency of the whole hierarchy is also verified. If the consistency condition is not satisfied, revisions of the pairwise comparisons are necessary for a trustworthy decision.

Hierarchy. The hierarchy has the general structure represented in Figure 8. The highest level represents the goal; the second level includes the criteria based on which the decision should be taken; the third level includes the possible alternatives which will be prioritised with respect to the criteria. The first step includes a decomposition of the decision problem into parts defined by all relevant attributes; these attributes are 


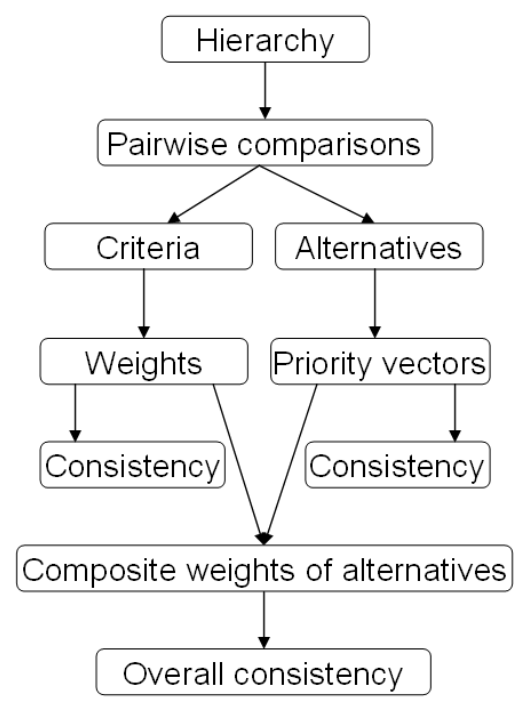

Figure 7: The Analytic Hierarchy Process.

arranged into hierarchical levels so as to reach the hierarchical structure presented in Figure 8 .

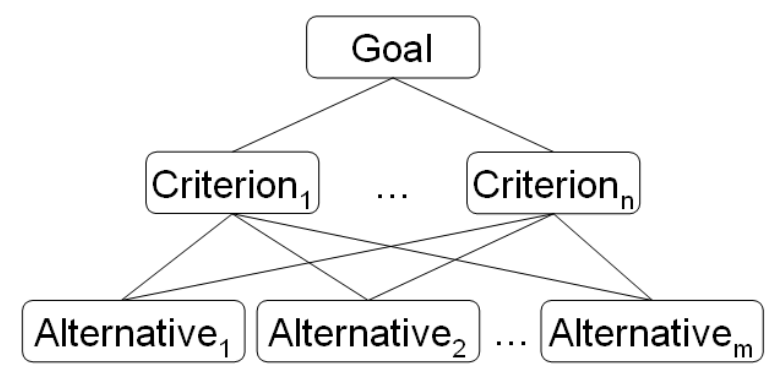

Figure 8: A hierarchy in the Analytic Hierarchy Process.

Priorities. The analysis of priorities includes pairwise comparisons used to compute weights for the alternatives; the weights will establish an order between the alternatives. This process involves two substeps: (a) decide the priorities between the criteria; (b) decide the priorities between alternatives with respect to each criterion. The priorities take the form of matrices (see Eq. (1)): one for the first substep, priorities amongst criteria, and $n$ for the second substep, priorities amongst alternatives (a matrix for each criterion). For both types of matrices the values of the lower diagonal part of the matrix are the inverse of the values of the upper diagonal part, i.e. $c_{j i}=1 / c_{i j}, a_{j i}=1 / a_{i j}$, as the comparison result between two objects $\mathrm{A}$ and $\mathrm{B}$ is reversed when the order changes, meaning between $\mathrm{B}$ and $\mathrm{A}$. 


$$
\left[\begin{array}{cccc}
1 & c_{12} & \ldots & c_{1 n} \\
1 / c_{12} & 1 & \ldots & c_{2 n} \\
\ldots & \ldots & \ldots & \ldots \\
1 / c_{1 n} & 1 / c_{2 n} & \ldots & 1
\end{array}\right]\left[\begin{array}{cccc}
1 & a_{12} & \ldots & a_{1 m} \\
1 / a_{12} & 1 & \ldots & a_{2 m} \\
\ldots & \ldots & \ldots & \ldots \\
1 / a_{1 m} & 1 / a_{2 m} & \ldots & 1
\end{array}\right]
$$

In Eq. (1), each pair of criteria $c_{i}$ and $c_{j}$ has an associated value that specifies their relative importance. The values of $c_{i j}(1 \leq i, j \leq n)$ and $a_{i j}(1 \leq i, j \leq m)$ are determined using a scale from 1 to 9 , where 1 means 'equally important' and 9 , means 'extremely more important'. For example, $c_{i j}=1$ means that the criteria $c_{i}$ and $c_{j}$ are equally important, $c_{i j}=3$ means that $c_{i}$ is more important than $c_{j}$ and $c_{i j}=9$ means that $c_{i}$ is extremely more important than $c_{j}$. The vales and meaning for the inverse pairs are: $c_{j i}=1: c_{j}$ and $c_{i}$ are equally important, $c_{j i}=1 / 3: c_{j}$ is less important than $c_{i}$ and $c_{j i}=1 / 9: c_{j}$ is extremely less important than $c_{i}$.

The weight of each criterion is calculated using Eq. (2) and the criteria weight vector is obtained: $W=\left(w_{1}, w_{2}, \ldots, w_{n}\right)$.

$$
w_{i}=\frac{\left(\prod_{j=1}^{n} c_{i j}\right)^{1 / n}}{\sum_{i=1}^{n}\left(\prod_{j=1}^{n} c_{i j}\right)^{1 / n}}
$$

For the alternatives, a priority vector is calculated for each matrix (corresponding to a criterion) using the same equation, i.e Eq. (2). Thus priority vectors: $A\left(C r_{j}\right)=$ $\left(A_{1}\left(C r_{j}\right), A_{2}\left(C r_{j}\right), \ldots, A_{m}\left(C r_{j}\right)\right), j=\overline{1, n}$ are obtained. The matrix A of Eq. (3) results from combining the $n$ priority vectors.

$$
A=\left[\begin{array}{cccc}
A_{1}\left(C r_{1}\right) & A_{1}\left(C r_{2}\right) & \cdots & A_{1}\left(C r_{n}\right) \\
A_{2}\left(C r_{1}\right) & A_{2}\left(C r_{2}\right) & \cdots & A_{2}\left(C r_{n}\right) \\
\vdots & \vdots & \ddots & \vdots \\
A_{m}\left(C r_{1}\right) & A_{m}\left(C r_{2}\right) & \cdots & A_{m}\left(C r_{n}\right)
\end{array}\right]
$$

By combining the criteria weights and the priority vectors the final alternatives priorities vector $P$ with respect to all criteria is obtained using Eq. (4). More specifically, the priority for each alternative is calculated as shown in Eq. (5).

$$
\begin{gathered}
P=A * W \\
p_{i}=A_{i}\left(C r_{1}\right) * w_{1}+A_{i}\left(C r_{2}\right) * w_{2}+\ldots+A_{i}\left(C r_{n}\right) * w_{n}, i=\overline{1, m}
\end{gathered}
$$

Consistency. To verify the consistency of the $n+1$ pairwise comparisons matrices ( $n$ alternatives matrices and 1 criteria matrix), an approximation of the maximum eigenvalue for each matrix, denoted as $\lambda_{\max }$ (see Eq. 6) is used to calculate the consistency index (CI). Eq. (7) shows how to calculate CI for the criteria matrix and the $n$ alternatives matrices. 


$$
\begin{array}{r}
\lambda_{\max _{j}}=\left(\sum_{i=1}^{m} a_{i 1}, \sum_{i=1}^{m} a_{i 2}, \ldots, \sum_{i=1}^{m} a_{i m}\right) * \\
\left(A_{1}\left(C r_{j}\right), A_{2}\left(C r_{j}\right), \ldots, A_{m}\left(C r_{j}\right)\right)^{T}, j=\overline{1, n}
\end{array}
$$

$$
\text { For criteria: } C I=\frac{\lambda_{\max }-n}{n-1}
$$

$$
\text { For alternatives: } C I_{j}=\frac{\lambda_{\max _{j}}-m}{m-1}, j=\overline{1, m}
$$

$\mathrm{CI}$ and the Random Consistency Index (RCI) are used to calculate the consistency ratio (CR) as in Eq. (8). The values of the RCI for 1 to 10 criteria are displayed in Table 7. Values of the consistency ratio below 0.10 indicate consistency, while greater values indicate the opposite. In the later case, revision of the pairwise comparisons is necessary.

$$
C R=\frac{C I}{R C I}
$$

Table 7: Values of RCI for $n=\overline{1,10}$.

\begin{tabular}{l|cccccccccc}
\hline $\mathrm{n}$ & 1 & 2 & 3 & 4 & 5 & 6 & 7 & 8 & 9 & 10 \\
\hline $\mathrm{RCI}$ & 0 & 0 & 0.58 & 0.90 & 1.12 & 1.24 & 1.32 & 1.41 & 1.45 & 1.49 \\
\hline
\end{tabular}

The overall consistency of the hierarchy is a function of the consistency indexes of all pairwise matrices, the RCI for the number of criteria and number of alternatives and the weights of the criteria, as in Eq. (9).

$$
C R=\frac{C I_{\text {criteria }}+w_{1} * C I_{a_{\text {alt }} \text { Cr }_{1}}+w_{2} * C I_{\text {alt }_{C r_{2}}}+\ldots+w_{n} * C I_{\text {alt }_{C r_{n}}}}{R C I_{n}+w_{1} * R C I_{m}+w_{2} * R C I_{m}+\ldots+w_{n} * R C I_{m}}
$$

Summarising, the AHP process involves three main steps: definition of the hierarchy, analysis of pairwise comparisons and verification of consistency. These are illustrated through an example in the following section.

\subsection{Scenario-based Validation of the MDM component}

One scenario is presented to illustrate the AHP process in the context of ShapeBuilder and tasks of the same type as pond-tiling. The hierarchy of the AHP formalism is illustrated in Figure 9: the goal is to obtain feedback priorities; the criteria is the type of context: specific and general; the alternatives are feedback on the following aspects: (a) correctness of construction (CC); (b) correctness of expression (CE); (c) constructionexpression correspondence (C-E); (d) symmetry of construction (Sym); (e) generality of construction (CGen); (f) generality of expression (EGen); (g) use of icon variables (IV). 


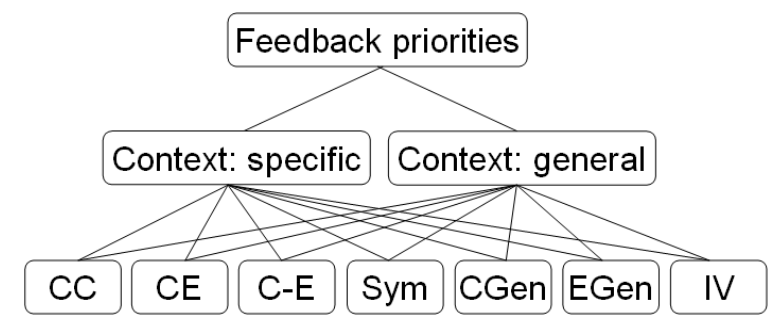

Figure 9: AHP hierarchy for prioritising alternative types of feedback.

The pairwise comparisons between criteria and between alternatives vary depending on learner's characteristics: (a) task difficulty; (b) level of experience (stored for each level of task difficulty) and (c) arithmetics knowledge level. To illustrate the application of AHP for prioritising feedback we consider a situation when the task difficulty is high, the level of experience of the learner with highly difficult tasks is low and his/her arithmetics knowledge level is good.

The pairwise comparisons and weights of the criteria are displayed in Table 8. As the learner is given a task of high difficulty only after having at least medium experience with low and medium task difficulty, the specific context is only slightly more important than the general one.

Table 8: Criteria pairwise comparisons and weights.

\begin{tabular}{c|cc|c}
\hline Criteria & Specific & General & Weights \\
\hline Specific & 1 & 2 & 0.67 \\
General & $1 / 2$ & 1 & 0.33 \\
\hline \multicolumn{4}{l}{$\lambda_{\max }=2, C I=0, C R=0$} \\
\hline
\end{tabular}

For each of the two criteria, i.e. specific and general context, there is a matrix of pairwise comparisons of the alternatives. The pairwise comparisons and the priority vector when the context is specific is displayed in Table 9, while the ones for the general context are displayed in Table 10. Both matrices are consistent.

Table 9: Alternatives pairwise comparisons and the priority vector with respect to specific context.

\begin{tabular}{l|ccccccc|c}
\hline Alternatives & CC & CE & C-E & Sym & CGen & EGen & IV & Priority Vector \\
\hline CC & 1 & 3 & 3 & 1 & 7 & 7 & 7 & 0.33 \\
CE & $1 / 3$ & 1 & $1 / 2$ & $1 / 2$ & 3 & 3 & 7 & 0.13 \\
C-E & $1 / 3$ & 2 & 1 & $1 / 2$ & 5 & 5 & 7 & 0.19 \\
Sym & 1 & 2 & 2 & 1 & 2 & 3 & 5 & 0.21 \\
CGen & $1 / 7$ & $1 / 3$ & $1 / 5$ & $1 / 2$ & 1 & 1 & $1 / 2$ & 0.04 \\
EGen & $1 / 7$ & $1 / 3$ & $1 / 5$ & $1 / 3$ & 1 & 1 & $1 / 3$ & 0.04 \\
IV & $1 / 7$ & $1 / 7$ & $1 / 7$ & $1 / 5$ & 2 & 3 & 1 & 0.05 \\
\hline \multicolumn{2}{l}{$\lambda_{\max }=7.52, C I=0.09, C R=0.07$} \\
\hline
\end{tabular}


Table 10: Alternatives pairwise comparisons and the priority vector with respect to general context.

\begin{tabular}{l|ccccccc|c}
\hline Alternatives & CC & CE & C-E & Sym & CGen & EGen & IV & Priority Vector \\
\hline CC & 1 & 1 & 1 & 1 & 2 & 2 & 1 & 0.16 \\
CE & 1 & 1 & $1 / 2$ & $1 / 2$ & $1 / 3$ & $1 / 3$ & $1 / 3$ & 0.07 \\
C-E & 1 & 2 & 1 & 1 & $1 / 3$ & $1 / 3$ & $1 / 3$ & 0.09 \\
Sym & 1 & 2 & 1 & 1 & $1 / 2$ & 2 & $1 / 3$ & 0.12 \\
CGen & $1 / 2$ & 3 & 3 & 2 & 1 & 2 & $1 / 2$ & 0.18 \\
EGen & $1 / 2$ & 3 & 3 & $1 / 2$ & $1 / 2$ & 1 & $1 / 2$ & 0.12 \\
IV & 1 & 3 & 3 & 3 & 2 & 2 & 1 & 0.26 \\
\hline \multicolumn{2}{l}{$\lambda_{\max }=7.62, C I=0.10, C R=0.08$}
\end{tabular}

The final alternatives priorities are displayed in Table 11. The most important aspect to give feedback on is the correctness of construction (CC), followed by symmetry (Sym), correspondence between construction and expression (C-E) and use of icon variables (IV); the next aspects to give feedback on are: correctness of expression (CE), construction generality (CGen) and expression generality (EGen).

Table 11: Feedback priorities.

\begin{tabular}{l|ccccccc}
\hline Alternatives & CC & CE & C-E & Sym & CGen & EGen & IV \\
\hline Priorities & 0.28 & 0.11 & 0.16 & 0.18 & 0.09 & 0.07 & 0.12 \\
\hline \multicolumn{7}{l}{ Overall $C R=0.03$} \\
\hline
\end{tabular}

Therefore, when the task difficulty is high, the learners' experience with this type of tasks is low and their arithmetics level is good, the most important aspect is the correctness of construction and its symmetry; symmetry is quite important as a generalisation of a difficult task is more challenging when the construction (and consequently the expression) is not symmetric; the correspondence between construction and expression is used in the development of the expression and is early encouraged so as to develop the expression in parallel to the construction; the use of icon variables is encouraged from the beginning in order to facilitate generalisation at a later stage. Hence, the focus is on the construction, guiding the learners to build it in a correct way, using symmetry and icon variables; the generality is addressed later, when these aspects are taken care of; also, the importance of the correspondence between construction and expression is stressed as to facilitate the derivation of the expression.

\section{Validating the CBR-AHP Synergy: an Integrative Sce- nario}

To illustrate the combination of the two intelligent methods, i.e. CBR and AHP, we use the "pond tiling" task discussed above. We will analyse the situation of two learners, Alan and Mike, who have built the same construction, but have different expressions; also, their learner models hold different values on their characteristics. 
We will start with the common aspect between the two: the construction. This is displayed in Figure 10; it is a combination of two strategies, 'I' and 'spiral', and consists of two 'general' cases $\left(C_{2}\right.$ and $\left.C_{3}\right)$ and two 'specific' ones $\left(C_{4}\right.$ and $\left.C_{5}\right)$. This is a symmetric situation and consequently, the comparison with the 'specific' and the 'general' strategies will give the same results. Moreover, because the surrounding has two cases from one strategy and two cases from the other one, when compared with these two strategies, the same result is obtained: $\frac{5}{\sqrt{8}}+\frac{3+4 / 3}{5}+\frac{3 / 4+1 / 3}{5}+\frac{10 / 4}{5}=3.35$. In such cases, as mentioned previously, there are several options for handling such a situation. A piece of information that may be important when deciding towards which strategy to guide the learner is related to the order in which the surrounding is done and the complexity of the strategies. In the current example, the learners start with a case from the 'spiral' strategy, but move to the 'I' strategy for the next two cases; to complete the surrounding, another case, which is typically encountered in the 'spiral' strategy, is used. The fact that 2 subsequent cases belong to the same strategy may be a good reason to guide the learner towards that particular strategy; however, it does not provide enough evidence on its own that learners' thinking of generalisation is coherent. Other actions of the learners and information stored in the learner models are necessary to reach an informed decision.

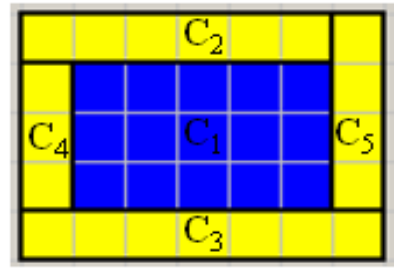

Figure 10: Combination of ' $\mathrm{I}$ ' and 'spiral' strategies $\left(C_{2}\right.$ and $C_{3}$ are general; $C_{4}$ and $C_{5}$ are specific).

The expressions built by Alan and Mike are displayed in Figure 11. Although Alan's construction has some generality (through the use of icon variables) the expression is still specific. Mike has some generality in the expression as well as the construction, though the two do not match.

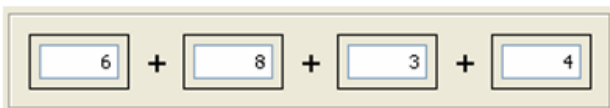

(a)

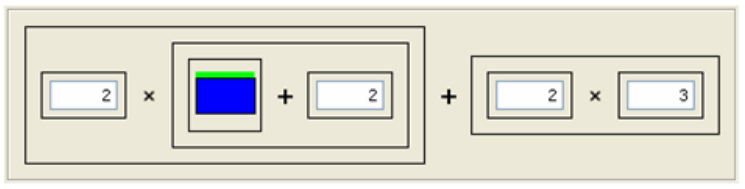

(b)

Figure 11: (a) Alan's expression (b) Mike's expression 
The difficulty of the "pond tiling" task is considered to be medium. On one hand, Alan has low experience with tasks of medium difficulty and a low level of arithmetic knowledge. On the other hand, Mike has high experience with medium difficulty tasks and his level of arithmetic knowledge is good. Taking these aspects into consideration and the context, i.e. 'specific' and 'general', the AHP is applied in order to find in which order the feedback on the different aspects (the seven alternatives presented in Section 6.2) should be provided.

For Alan, the pairwise comparisons and the weights for the criteria (the two types of context) are displayed in Table 12. As his experience with medium difficulty tasks is low, the 'specific' context criterion is more important than the 'general' context one. The matrices for the alternatives are displayed in Table 13 and Table 14: the first for the 'specific' criterion and the second for the 'general' one. The final alternatives priorities are displayed in Table 15.

Table 12: Alan: Criteria pairwise comparisons and weights.

\begin{tabular}{c|cc|c}
\hline Criteria & Specific & General & Weights \\
\hline Specific & 1 & 3 & 0.75 \\
General & $1 / 3$ & 1 & 0.25 \\
\hline \multicolumn{4}{l}{$\lambda_{\max }=2.00, C I=0, C R=0$} \\
\hline
\end{tabular}

Table 13: Alan: Alternatives pairwise comparisons and the priority vector with respect to specific context.

\begin{tabular}{l|ccccccc|c}
\hline Alternatives & CC & CE & C-E & Sym & CGen & EGen & IV & Priority Vector \\
\hline CC & 1 & 5 & 5 & 3 & 9 & 9 & 9 & 0.44 \\
CE & $1 / 5$ & 1 & $1 / 2$ & 3 & 5 & 5 & 9 & 0.17 \\
C-E & $1 / 5$ & 2 & 1 & 3 & 5 & 5 & 9 & 0.21 \\
Sym & $1 / 3$ & $1 / 3$ & $1 / 3$ & 1 & $1 / 3$ & 3 & $1 / 7$ & 0.04 \\
CGen & $1 / 9$ & $1 / 5$ & $1 / 5$ & 3 & 1 & 1 & $1 / 2$ & 0.05 \\
EGen & $1 / 9$ & $1 / 5$ & $1 / 5$ & $1 / 3$ & 1 & 1 & $1 / 5$ & 0.03 \\
IV & $1 / 9$ & $1 / 9$ & $1 / 9$ & 7 & 2 & 5 & 1 & 0.07 \\
\hline \multicolumn{2}{l|}{$\lambda_{\max }=8.62, C I=0.27, C R=0.20$} & \multicolumn{10}{l}{} \\
\hline
\end{tabular}

Table 14: Alan: Alternatives pairwise comparisons and the priority vector with respect to general context.

\begin{tabular}{l|ccccccc|c}
\hline Alternatives & CC & CE & C-E & Sym & CGen & EGen & IV & Priority Vector \\
\hline CC & 1 & 1 & 1 & 2 & 2 & 2 & 1 & 0.16 \\
CE & 1 & 1 & 1 & 2 & $1 / 5$ & $1 / 7$ & $1 / 5$ & 0.06 \\
C-E & 1 & 1 & 1 & 2 & $1 / 5$ & $1 / 7$ & $1 / 5$ & 0.06 \\
Sym & $1 / 2$ & $1 / 2$ & $1 / 2$ & 1 & $1 / 3$ & $1 / 3$ & $1 / 7$ & 0.05 \\
CGen & $1 / 2$ & 5 & 5 & 3 & 1 & 2 & $1 / 2$ & 0.20 \\
EGen & $1 / 2$ & 7 & 7 & 3 & $1 / 2$ & 1 & $1 / 2$ & 0.18 \\
IV & 1 & 5 & 5 & 7 & 2 & 2 & 1 & 0.30 \\
\hline
\end{tabular}


Table 15: Alan: Feedback priorities.

\begin{tabular}{l|ccccccc}
\hline Alternatives & CC & CE & C-E & Sym & CGen & EGen & IV \\
\hline Priorities & 0.37 & 0.14 & 0.17 & 0.04 & 0.08 & 0.07 & 0.12 \\
\hline \multicolumn{2}{l}{ Overall $C R=0.07$} \\
\hline
\end{tabular}

The two matrices of pairwise comparisons between alternatives are not consistent according to the standard measurement of consistency, i.e. $C R$, although the overall hierarchy is consistent $(C R=0.07)$. The revised alternatives matrices are displayed in Table 16 and Table 17. The new final priorities are displayed in Table 18.

Table 16: Alan: Alternatives pairwise comparisons and the priority vector with respect to specific context - revised.

\begin{tabular}{l|ccccccc|c}
\hline Alternatives & CC & CE & C-E & Sym & CGen & EGen & IV & Priority Vector \\
\hline CC & 1 & 4 & 4 & 5 & 9 & 9 & 9 & 0.44 \\
CE & $1 / 4$ & 1 & $1 / 2$ & 3 & 5 & 5 & 7 & 0.17 \\
C-E & $1 / 4$ & 2 & 1 & 5 & 5 & 5 & 7 & 0.22 \\
Sym & $1 / 5$ & $1 / 3$ & $1 / 5$ & 1 & $1 / 2$ & 2 & $1 / 5$ & 0.04 \\
CGen & $1 / 9$ & $1 / 5$ & $1 / 5$ & 2 & 1 & 1 & $1 / 2$ & 0.04 \\
EGen & $1 / 9$ & $1 / 5$ & $1 / 5$ & $1 / 2$ & 1 & 1 & $1 / 3$ & 0.03 \\
IV & $1 / 9$ & $1 / 7$ & $1 / 7$ & 5 & 2 & 3 & 1 & 0.06 \\
\hline \multicolumn{2}{l|}{$\lambda_{\max }=7.81, C I=0.14, C R=0.10$} & & \multicolumn{1}{l}{} \\
\hline
\end{tabular}

Table 17: Alan: Alternatives pairwise comparisons and the priority vector with respect to general context - revised.

\begin{tabular}{l|ccccccc|c}
\hline Alternatives & CC & CE & C-E & Sym & CGen & EGen & IV & Priority Vector \\
\hline CC & 1 & 1 & 1 & 2 & 2 & 2 & 1 & 0.18 \\
CE & 1 & 1 & 1 & 2 & $1 / 3$ & $1 / 3$ & $1 / 3$ & 0.09 \\
C-E & 1 & 1 & 1 & 2 & $1 / 3$ & $1 / 3$ & $1 / 3$ & 0.09 \\
Sym & $1 / 2$ & $1 / 2$ & $1 / 2$ & 1 & $1 / 2$ & $1 / 2$ & $1 / 3$ & 0.07 \\
CGen & $1 / 2$ & 3 & 3 & 2 & 1 & 2 & $1 / 2$ & 0.18 \\
EGen & $1 / 2$ & 3 & 3 & 2 & $1 / 2$ & 1 & $1 / 2$ & 0.15 \\
IV & 1 & 3 & 3 & 3 & 2 & 2 & 1 & 0.25 \\
\hline \multicolumn{2}{l|}{$=0.09, C R=0.07$} \\
\hline
\end{tabular}

Table 18: Alan: Feedback priorities - revised.

\begin{tabular}{l|ccccccc}
\hline Alternatives & CC & CE & C-E & Sym & CGen & EGen & IV \\
\hline Priorities & 0.37 & 0.15 & 0.19 & 0.05 & 0.08 & 0.06 & 0.11 \\
\hline \multicolumn{2}{l}{ Overall $C R=0.04$} \\
\hline
\end{tabular}

The final order of alternatives is the same in the revised version as the first (apparently) inconsistent one, although there are small differences in the values. The revised 
matrices were obtained using Saaty's suggested method of reconsidering the alternatives for which the corresponding rows in the matrix $\left[\left|a_{i j}-\left(p_{i} / p j\right)\right|\right]$ have the largest sums. Looking at the original and the new matrices, a change in numbers is observed, but with the preservation of the transitivity between the alternatives. As the idea is to prioritise between alternatives, transitivity is a logical condition and maybe the only necessary indication of consistency, as the preservation of priorities order seems to indicate.

Coming back to the educational meaning of these priorities, for Alan, the most important aspect for feedback is the correctness of construction, followed by the correspondence between construction and expression, and the correctness of expression. Thus, as Alan's arithmetic knowledge is low, his correct construction (already achieved, although not symmetric) and its correspondence with the expression (partial correspondence) lead Alan towards a correct expression. The following aspects to give feedback on are: (a) the use of icon variables - thus, Alan will be encouraged to introduce the icon variables in his expression and also to use the icon variable for the tiles corresponding to cases $C_{4}$ and $C_{5}$; (b) the generality of the construction, which can be achieved through using icon variables; (c) expression's generality and (d) symmetry. Thus, Alan will be guided to achieve a correct and general construction and expression before introducing symmetry. After this has been achieved, the benefits of symmetry will be presented and Alan will be guided to the 'spiral' or 'I' strategy through one of the options presented in Section 5.3.2. Letting the learner choose is not a feasible option, as Alan has low experience with this type of tasks; if there would be an automatic choice between the two, Alan would probably be guided towards the 'I' strategy; alternatively, the teacher could be informed about Alan's activity and she/he could choose one of the two.

The pairwise comparisons and weights of the criteria for Mike are displayed in Table 19. As Mike's level of experience with medium difficulty tasks is high, the 'general' context criterion is significantly more important than the 'specific' context one. The alternatives matrices are displayed in Table 20 for the 'specific' context criterion and Table 21 for the 'general' context criterion. The final alternatives priorities for Mike are displayed in Table 22. All matrices are consistent.

Table 19: Mike: Criteria pairwise comparisons and weights.

\begin{tabular}{c|cc|c}
\hline Criteria & Specific & General & Weights \\
\hline Specific & 1 & $1 / 5$ & 0.17 \\
General & 5 & 1 & 0.83 \\
\hline \multicolumn{4}{c}{$\lambda_{\max }=2.00, C I=0, C R=0$} \\
\hline
\end{tabular}

For Mike, the most important aspect for feedback is the use of icon variables, aiming to introduce generality early in the process of solving the task, as Mike has high experience with similar tasks (from difficulty point of view). Thus, Mike will be encouraged to use icon variables for the tiles corresponding to cases $C_{4}$ and $C_{5}$. The second important aspect, construction generality, is done using the icon variables and only after that feedback should address the correctness of construction. Thus, in contrast to what happened with Alan, Mike is first guided towards 'thinking in a general 
Table 20: Mike: Alternatives pairwise comparisons and the priority vector with respect to specific context.

\begin{tabular}{l|ccccccc|c}
\hline Alternatives & CC & CE & C-E & Sym & CGen & EGen & IV & Priority Vector \\
\hline CC & 1 & 3 & 3 & 2 & 5 & 5 & 5 & 0.35 \\
CE & $1 / 3$ & 1 & $1 / 2$ & 1 & 3 & 3 & 5 & 0.15 \\
C-E & $1 / 3$ & 2 & 1 & $1 / 2$ & 3 & 3 & 5 & 0.17 \\
Sym & $1 / 2$ & 1 & $1 / 2$ & 1 & 3 & 3 & 5 & 0.16 \\
CGen & $1 / 5$ & $1 / 3$ & $1 / 3$ & $1 / 3$ & 1 & 1 & $1 / 3$ & 0.05 \\
EGen & $1 / 5$ & $1 / 3$ & $1 / 3$ & $1 / 3$ & 1 & 1 & $1 / 7$ & 0.04 \\
IV & $1 / 5$ & $1 / 5$ & $1 / 5$ & $1 / 5$ & 3 & 7 & 1 & 0.07 \\
\hline & \multicolumn{7}{l}{$\lambda_{\max }=7.51, C I=0.09, C R=0.06$} \\
\hline
\end{tabular}

Table 21: Mike: Alternatives pairwise comparisons and the priority vector with respect to general context.

\begin{tabular}{l|ccccccc|c}
\hline Alternatives & CC & CE & C-E & Sym & CGen & EGen & IV & Priority Vector \\
\hline CC & 1 & 1 & 1 & 2 & $1 / 2$ & 1 & 1 & 0.13 \\
CE & 1 & 1 & $1 / 2$ & $1 / 2$ & $1 / 3$ & $1 / 3$ & $1 / 3$ & 0.07 \\
C-E & 1 & 2 & 1 & $1 / 2$ & $1 / 3$ & $1 / 3$ & $1 / 3$ & 0.08 \\
Sym & $1 / 2$ & 2 & 2 & 1 & 1 & 3 & $1 / 3$ & 0.14 \\
CGen & 2 & 3 & 3 & 1 & 1 & 2 & 1 & 0.22 \\
EGen & 1 & 3 & 3 & $1 / 3$ & $1 / 2$ & 1 & $1 / 3$ & 0.12 \\
IV & 1 & 3 & 3 & 3 & 1 & 3 & 1 & 0.24 \\
\hline
\end{tabular}

Table 22: Mike: Feedback priorities.

\begin{tabular}{l|ccccccc}
\hline Alternatives & CC & CE & C-E & Sym & CGen & EGen & IV \\
\hline Priorities & 0.17 & 0.08 & 0.10 & 0.15 & 0.19 & 0.11 & 0.21 \\
\hline \multicolumn{2}{l}{ Overall $C R=0.03$} \\
\hline
\end{tabular}

way' and then 'fixing' the construction/expression. Symmetry is then considered as the next important aspect of a construction because it facilitates generalisation and the construction of the corresponding general expression, which follows afterwards. At this point the eventual mismatch between the construction and the expression are addressed and, through this, Mike is guided towards a correct expression. Although Mike's expression did not correspond to his construction, this aspect is not addressed immediately; also, the expression shows a way of solving the task that is symmetric, which will probably be reflected in the construction later on.

Table 23 summarises this integrative scenario by presenting the various pieces of information that are combined, including the input from the Task LTM, which stores inferences of the CBR component, and the Domain LTM, and the output from the Feedback Priorities component. Thus, a similar diagnosis of the learners' models obtained by the CBR component would lead to different outputs of the AHP component depending on extra information available about the learners. 
Table 23: Feedback priorities depending on the learners' model data.

\begin{tabular}{|c|c|c|c|}
\hline & & Alan & Mike \\
\hline \multirow{5}{*}{$\begin{array}{l}\text { Task } \\
\text { LTM }\end{array}$} & task & pond-tiling & pond-tiling \\
\hline & difficulty & medium & medium \\
\hline & strategies & $\begin{array}{l}\text { 'I' } \\
\text { 'spiral' }\end{array}$ & $\begin{array}{l}\text { 'I' } \\
\text { 'spiral' }\end{array}$ \\
\hline & \multirow[t]{2}{*}{ expression } & & \multirow{2}{*}{$\square \times \square+\square+\square \times \square$} \\
\hline & & \begin{tabular}{|l}
$\square$ \\
8
\end{tabular} & \\
\hline \multirow{2}{*}{$\begin{array}{l}\text { Domain } \\
\text { LTM }\end{array}$} & experience & low & high \\
\hline & arithmetics level & low & good \\
\hline \multicolumn{2}{|c|}{ Feedback priorities } & $\begin{array}{l}\text { CC, C-E, CE, IV, } \\
\text { CGen, EGen, Sym }\end{array}$ & $\begin{array}{l}\text { IV, CGen, CC, Sym } \\
\text { EGen, C-E, CE }\end{array}$ \\
\hline
\end{tabular}

\section{Discussion and Conclusions}

In this paper we presented a synergistic approach for learner modelling and feedback aiming to solve some challenges encountered in exploratory learning environments. This work addresses the necessity of feedback in ELEs during rather than at the end of a task and of feedback prioritisation as exploratory activities often require feedback on several aspects. The learner modelling framework we presented aims to support the provision of feedback tailored to the learners through a detailed and frequently updated learner model. We illustrated this approach in ShapeBuilder, an exploratory learning environment for mathematical generalisation.

The core of our approach is a hybrid model that combines the advantages of representation flexibility from case-based reasoning, and prioritisation from multicriteria decision making, when several aspects need to be accounted for. A representation of the tasks is stored in a case-base forming a Task Model. Models produced by the learners are compared against these cases to identify misconceptions and underlying strategies that require support. This information is further used to prioritise the aspects that require feedback depending on the learners' long-term characteristics.

One of the challenges encountered when building the task knowledge base was the representation of the task in a way that facilitates diagnosis and monitoring of what the learners are building in the system. The current case-based representation was chosen for its flexibility, despite the fact that it does not 'fit' into the standard representation used in CBR, where a case usually represents a problem with its definition and solution. In our approach, we use simple and composite cases, i.e. strategies (build up from simple cases); the latter representing potential problem solutions. This allows the diagnosis of the learners constructions while they are building them.

The cooperative inquiry design methodology adopted in this work involved several iterations and refinements of the software, consequently taking longer than other approaches to software development. However, the participatory and iterative methodology captured the way children interact with the system and thus, informed the development of the learner modelling and feedback components. 
Another practical issue related to the CBR approach is scalability. The scenarios presented covered some of the most complex situations encountered in the trials with pupils, suggesting therefore, that this approach will scale-up when the complexity of the learners' constructions increases.

Another considerable challenge was to frame the feedback priorities problem in terms of the AHP. The examples provided in this paper were limited to feedback on model and expression construction. However, there are other aspects to give feedback on [42], like setting goals, organising the working environment, supporting reflection and collaboration, in which case the hierarchy becomes more complex. These aspects will be addressed in future work together with an evaluation of this approach in a real classroom setting.

\section{References}

[1] V. Aleven, 'Using background knowledge in case-based legal reasoning: A computational model and an intelligent learning environment', Artificial Intelligence, 150(1-2), 183-237, (2003).

[2] J.E. Beck and B.P. Woolf, 'Using a Learning Agent with a Student Model', in Intelligent Tutoring Systems, eds., B.P. Goettl, H.M. Halff, C.L. Redfield, and V.J. Shute, volume 1452 of Lecture Notes in Computer Science, pp. 6-15. Springer, (1998).

[3] P. Brusilovsky, A.T. Corbett, and F. de Rosis, eds. User Modeling 2003, 9th International Conference, UM 2003, Johnstown, PA, USA, June 22-26, 2003, Proceedings, volume 2702 of Lecture Notes in Computer Science. Springer, 2003.

[4] A. Bunt and C. Conati, 'Probabilistic Student Modelling to Improve Exploratory Behaviour', User Modelling and User-Adaptive Interaction, 13(3), 269-309, (2003).

[5] F.T.S. Chan, 'Application of a hybrid case-based reasoning approach in electtroplating industry', Expert Systems with Applications, 29(1), 121-130, (2005).

[6] F.T.S. Chan, S.H. Chung, and K.L. Choy, 'Optimisation of order fulfillment in distribution netwrok problems', Journal of Intelligent Manufactoring, 17(3), 307$319,(2006)$.

[7] P-C. Chang, C-H. Liu, and R.K. Lai, 'A fuzzy case-based reasoning model for sales forecasting in print circuit board industries', Expert Systems with Applications, 34(3), 2049-2058, (2008).

[8] S-H. Chun and Y-J. Park, 'Dynamic adaptive ensemble case-based reasoning: application to stock market prediction', Expert Systems with Application, 28(3), 435-443, (2005).

[9] S-H. Chun and Y-J. Park, 'A new hybrid data mining technique using a regression case based reasoning: Application to financial forecasting', Expert Systems with Applications, 31(2), 329-336, (2006). 
[10] C. Conati, A.S. Gertner, and K. VanLehn, 'Using Bayesian Networks to Manage Uncertainty in Student Modeling', User Modelling and User-Adaptive Interaction, 12(4), 371-417, (2002).

[11] M. Crary, L.K. Nozick, and L.R. Whitaker, 'Sizing the us destroyer fleet', European Journal of Operational Research, 136(3), 680-695, (2002).

[12] A. Druin, 'The role of children in the design of new technology', Behaviour and Information Technology, 21(1), 1-25, (2002).

[13] T. Ertay, D. Ruan, and U.R. Tuzkaya, 'Integrating data envelopment analysis and analytic hierarchy for the facility layout design in manufacturing systems', Information Sciences, 176(3), 237-262, (2006).

[14] G. Ghinea, G.D. Magoulas, and C. Siamitros, 'Multicriteria decision making for enhanced perception-based multimedia communication', IEEE Transactions on Systems, Man, and Cybernetics, Part A, 35(6), 855-866, (2005).

[15] J. Good and J. Robertson, 'Carss: A framework for learner-centred design with children', International Journal of Artificial Intelligence in Education, 16, 381413, (2006).

[16] M. Grigoriadou, H. Kornilakis, K.A. Papanikolaou, and G.D. Magoulas, 'Fuzzy inference for student diagnosis in adaptive educational systems', in Methods and Applications of Artificial Intelligence: Proceedings of the 2nd Hellenic Conference on AI, SETN2002, eds., I.P. Vlahavas and C.D. Spyropoulos, volume 2308 of Lecture Notes in Artificial Intelligence, pp. 191-202. Berlin: Springer-Verlag, (2002).

[17] S. Gutiérrez, M. Mavrikis, and D. Pierce, 'A Learning Environment for Promoting Structured Algebraic Thinking in Children', in Proceedings of the 8th IEEE International Conference on Advanced Learning Technologies, pp. 74-76, (2008).

[18] S. Gutiérrez, D. Pearce, E. Geraniou, and M. Mavrikis, 'Supporting reasoning and problem-solving in mathematical generalisation with dependency graphs', in Diagrams, eds., G. Stapleton, J. Howse, and J. Lee, volume 5223 of Lecture Notes in Computer Science, pp. 374-377. Springer, (2008).

[19] S-G. Han, S-G. Lee, and S. Jo, 'Case-based tutoring systems for procedural problem solving on the www', Expert Systems with Applications, 29(3), 573-582, (2005).

[20] I. Hatzilygeroudis and J. Prentzas, 'Using a hybrid rule-based approach in developing an intelligent tutoring system with knowledge acquisition and update capabilities', Expert Systems with Applications, 26(4), 477-492, (2004).

[21] L. Healy, Hoelzl R., Hoyles C., and R. Noss, 'Messing Up', Micromath, 10(1), 14-16, (1994). 
[22] W. Ho, 'Integrated analytic hierarchy process and its applications a literature review', European Journal of Operational Research, 186(1), 211-228, (2008).

[23] C-C. Hsu and C-S. Ho, 'A new hybrid case-based architecture for medical diagnosis', Infomation Sciences, 166(1-4), 231-247, (2004).

[24] M-J. Huang, H-S. Huang, and M-Y. Chen, 'Constructing a personalized elearning system based on genetic algorithm and case-based reasoning approach', Expert Systems with Applications, 33(3), 551-564, (2007).

[25] C.D. Hulshof, T.H.S. Eysink, S. Loyens, and T. de Jong, 'Zaps: Using interactive programs for learning psychology', Interactive Learning Environments, 13(1-2), 39-53, (2005).

[26] J. Kaput, Technology and Mathematics education, 515-556, D. Grouws (ed.) Handbook of Research on Mathematics Teaching and Learning, New York: Macmillan, 1992.

[27] P. Kirschner, J. Sweller, and R.E. Clark, 'Why minimal guidance during instruction does not work: An analysis of the failure of constructivist, discovery, problem-based, experiential and inquiry-based teaching', Educational Psychologist, 41(2), 75-86, (2006).

[28] G. Koksal and A. Egitman, 'Planning and design od industrial engineering education quality', Computers and Industrial Engineering, 35(3-4), 639-642, (1998).

[29] J.L. Kolodner, Case-Based Reasoning, Morgan Kaufmann Publishers, Inc., 2nd edn., 1993.

[30] I. Korkmaz, Gkcen H., and T. Çetinyokus, 'An analytic hierarchy process and two-sided matching based decision support system for military personnel assignment', Information Sciences, 178(14), 2915-2927, (2008).

[31] C. Koutsojannis, G. Beligiannis, I. Hatzilygeroudis, C. Papavlasopoulos, and J. Prentzas, 'Using a hybrid ai approach for exercise difficulty level adaptation', International Journal of Continuing Engineering Education and Life-Long Learning, 17(4-5), 256-272, (2007).

[32] P. Kumar, S. Gopalan, and V. Sridhar, 'Context enabled multi-CBR based recommendation engine for e-commerce', in Proceedings of the IEEE International Conference on e-Business Engineering (ICEBE), pp. 237 - 244. IEEE Press, (2005).

[33] N.K. Kwak and C.W. Lee, 'A multicriteria decision-making approach to university resource allocation and information infrastructure planning', European Journal of Operational Research, 110(2), 234-242, (1998).

[34] N.K. Kwak a and C.W. Lee, 'Business process reengineering for health-care system using multicriteria mathematical programming', European Journal of Operational Research, 140(2), 447-458, (2002). 
[35] O. Kwon and M. Kim, 'Mymessage: case-based reasoning and multicriteria decision making techniques for intelligent context-aware message filtering', Expert Systems with Applications, 27(3), 467-480, (2004).

[36] K. Lam and X. Zhao, 'An application of quality function deployment to improve the quality of teaching', International Journal of Quality and Reliability Management, 15(4), 389-413, (1998).

[37] C.W. Lee and N. K. Kwak, 'Information resource planning for a health-care system using an ahp-based goal programming method', Journal of Operational Research Society, 50(12), 1191-1198, (1999).

[38] G.H. Lee, 'Rule-based and case-based reasoning approach for internal audit of bank', Knowledge-Based Systems, 21(2), 140-147, (2008).

[39] C-H. Liu, L-S. Chen, and C-C. Hsu, 'An association-based case reduction technique for case-based reasoning', Information Sciences, 178(17), 3347-3355, (2008).

[40] I. Lopez-Arevalo, R. Banares-Alcantara, A. Aldea, A. Rodriguez-Martinez, and L. Jimenez, 'Generation of process alternatives using abstract models and casebased reasoning', Computers and Chemical Engineering, 31(8), 902-918, (2007).

[41] J. Mason, Generalisation and algebra: Exploiting children's powers, 105-120, L. Haggarty, Aspects of Teaching Secondary Mathematics: Perspectives on Practice, Routledge Falmer and the Open University, 2002.

[42] M. Mavrikis, E. Geraniou, R. Noss, and C. Hoyles, 'Revisiting pedagogic strategies for supporting students learning in mathematical microworlds. devolving teachers role to an intelligent facilitator', in ISEE workshop, eds., S. Gutiérrez and M. Mavrikis, pp. 41-50, (2008).

[43] R. Mayer, 'Should There Be a Three-Strikes Rule Against Pure Discovery Learning? The Case for Guided Methods of Instruction', American Psychologist, 59(1), 14-19, (2004).

[44] R. Morales Gamboa, Exploring Participative Learner Modelling and Its Effects on Learner Behaviour, $\mathrm{PhD}$ thesis, Univ. of Edinburgh, 2000.

[45] M.S. Ozdemir and R. N. Gasimov, 'The analytic hierarchy process and multiobjective 01 faculty course assignment', European Journal of Operational Research, 157(2), 398-408, (2004).

[46] R. Pan, Q. Yang, and Pan S.J., 'Mining competent case bases for case-based reasoning', Artificial Intelligence, 171(16-17), 1039-1068, (2007).

[47] C-S. Park and I. Han, 'A case-based reasoning with the feature weights derived by analytic hierarchy process for bankruptcy prediction', Expert Systems with Applications, 23(1), 255-264, (2002). 
[48] J.H. Park, K.H. Im, C-K. Shin, and S-C. Park, 'MBNR: Case-Based Reasoning with Local Feature Weighting by Neural Network', Applied Intelligence, 21(3), 265-276, (2004).

[49] F. Y. Partovi, 'An analytic model for locating facilities strategically', Omega, 34(1), 41-55, (2006).

[50] D. Pearce, M. Mavrikis, E. Geraniou, and S. Gutiérrez, 'Issues in the design of an environment to support the learning of mathematical generalisation', in EC-TEL, eds., P. Dillenbourg and M. Specht, volume 5192 of Lecture Notes in Computer Science, pp. 326-337. Springer, (2008).

[51] C. Romero, S. Ventura, P. de Bra, and C. de Castro, 'Discovering Prediction Rules in AHA! Courses', In Brusilovsky et al. [3], pp. 25-34.

[52] T.L. Saaty, The Analytic Hierarchy Process, New York: McGraw-Hill, 1980.

[53] C. Schmitt, D. Dengler, and M. Bauer, 'Multivariate preference models and decision making with the maut machine.', in User Modeling, eds., P. Brusilovsky, A. Corbett, and F. de Rosis, volume 2702 of Lecture Notes in Artificial Intelligence, pp. 297-302. Springer, (2003).

[54] Y. Seo, D. Sheen, and T. Kim, 'Block assembly planning in shipbuilding using case-based reasoning', Expert Systems with Applications, 32(1), 245-253, (2007).

[55] Z. Shen, H.C. Lui, and L. Ding, 'Approximate case-based reasoning on neural networks', International Journal of Approximate reasoning, 10(1), 75-98, (1994).

[56] H. Shinno, H. Yoshioka, S. Marpaung, and S. Hachiga, 'Quantitative swot analysis on global competitiveness of machine tool industry', Journal of Engineering Design, 17(3), 251-258, (2006).

[57] R. Stathacopoulou, M. Grigoriadou, G.D. Magoulas, and D. Mitropoulos, 'A Neuro-fuzzy Approach in Student Modeling', In Brusilovsky et al. [3], pp. 337341.

[58] R.E. Steuer and P. Na, 'Multicriteria decision making combined with finance: A categorized bibliographic study', European Journal of Operational Research, 150(3), 496-515, (2003).

[59] R.H. Stottler and S. Ramachandran, 'A Case-Based Reasoning Approach to Internet Intelligent Tutoring Systems (ITS) and ITS Authoring', in Proceedings of the Twelfth International Florida Artificial Intelligence Research Society Conference, pp. 181-186. AAAI Press, (1999).

[60] J. Swaak, W.R. van Joolingen, and T. de Jong, 'Supporting simulation-based learning; the effects of model progression and assignments on definitional and intuitive knowledge', Learning and Instruction, 8(3), 235-253, (1998).

[61] O.S. Vaidya and S. Kumar, 'Analytic hierarchy process: An overview of applications', European Journal of Operational Research, 169(1), 1-29, (2006). 
[62] W.R. van Joolingen, 'Cognitive tools for discovery learning', International Journal of Artificial Intelligence and Education, 10, 385-397, (1999).

[63] P. Wriggers, M. Siplivaya, I. Joukova, and R. Slivin, 'Intelligent support of engineering analysis using ontology and case-base reasoning', Engineering Applications of Artificial Intelligence, 20(5), 709-720, (2007).

[64] R.R. Yager, 'Soft Aggregation Methods in Case Based Reasoning', Applied Intelligence, 21(3), 277-288, (2004).

[65] H-L. Yang and C-S. Wang, 'Two stages of case-based reasoning - integrating genetic algorithm with data mining mechanism', Expert Systems with Applications, 35(1-2), 262-272, (2008).

[66] C. Zopounidis and M. Doumpos, 'Multicriteria classification and sorting methods: A literature review', European Journal of Operational Research, 138(2), 229-246, (2002). 